\title{
AUTOMORPHISMS FOR THE LOGARITHMIC PRODUCT OF POSITIVE SEMIDEFINITE OPERATORS
}

\author{
GREGOR DOLINAR AND LAJOS MOLNÁR
}

\begin{abstract}
In this paper we consider the set of all positive semidefinite linear operators on a finite dimensional Hilbert space equipped with the so-called logarithmic product. We describe the general form of all automorphisms of this structure which are continuous at 0 .
\end{abstract}

Let $H$ be a complex Hilbert space of finite dimension. We denote by $B(H)$ the algebra of all linear operators on $H$, and $B_{s}(H), B(H)^{+}$stand for the linear space of all self-adjoint elements and the cone of all positive semidefinite elements of $B(H)$, respectively. The collection of all invertible elements of $B(H)^{+}$, i.e., the set of all positive definite operators on $H$ is denoted by $B(H)_{-1}^{+}$. This space equipped with different algebraic or analytical structures plays important roles in several branches of mathematics.

The so-called logarithmic product $A \odot B$ of positive definite operators $A, B \in B(H)_{-1}^{+}$is defined by

$$
A \odot B=\exp (\log A+\log B) .
$$

This operation has turned to have serious applications in computer graphics and medical imaging with DT-MRI and hence it has recently been studied in details in the literature, see e.g., [1], [2], [3]. In the latter paper the authors have shown that the logarithmic product makes the set $B(H)_{-1}^{+}$a commutative Lie group and also explored a natural metric compatible with that structure. This is called log-Euclidean metric and it is defined by

$$
d(A, B)=\sqrt{\operatorname{tr}\left((\log A-\log B)^{2}\right)} \quad\left(A, B \in B(H)_{-1}^{+}\right),
$$

where $\operatorname{tr}$ is the usual trace functional. In the recent paper [8], Warmuth and Kuzmin have extended the definition of the logarithmic product for arbitrary positive semidefinite operators and presented its applications in

1991 Mathematics Subject Classification. Primary: 47B49. Secondary: 15A30, 08A35.

Key words and phrases. Positive semidefinite operators, logarithmic product, automorphisms.

The first author was supported by a grant from the Ministry of Higher Education, Science and Technology, Slovenia. The second author was supported by the Alexander von Humboldt Foundation (Germany), by the Hungarian Scientific Research Fund (OTKA) K81166 NK81402, and by the TÁMOP 4.2.1./B-09/1/KONV-2010-0007 project implemented through the New Hungary Development Plan co-financed by the European Social Fund and the European Regional Development Fund. 
quantum physics. Namely, they have used this product to define conditional density matrices and then obtained generalizations of quantum Bayes rule.

As seen, the set $B(H)^{+}$equipped with the logarithmic product is an interesting algebraic structure that draws attention from different points of view. To mention only one surprising fact: $\odot$ is an associative and commutative(!) operation which equals the usual operator product for commuting operators. In the present paper we contribute to the study of this semigroup by describing the structure of its automorphisms under a mild continuity assumption.

First observe that the study of the $\odot$-automorphisms of the Lie group $B(H)_{-1}^{+}$is not of real interest. Indeed, those automorphisms of $B(H)_{-1}^{+}$ are in a one-to-one correspondence with the additive bijections of $B_{s}(H)$. To show this, for any $\odot$-automorphism $\phi$ of $B(H)_{-1}^{+}$consider the transformation $\psi=\log \circ \phi \circ \exp$ on $B_{s}(H)$ which is easily seen to be merely a bijective additive map. Even if we assume that $\phi$ is continuous with respect to, say, the log-Euclidean metric (which means precisely that the transformation $\psi=\log \circ \phi \circ \exp$ is continuous with respect to the Hilbert-Schmidt norm (Euclidean metric) on $B_{s}(H)$ ), this gives no more than $\psi$ is a bijective linear transformation on $B_{s}(H)$. Hence, the group of all continuous automorphisms of $B(H)_{-1}^{+}$is isomorphic to the group of all nonsingular linear transformations of $B_{s}(H)$. The same conclusion holds if we change the logEuclidean topology to the topology on $B(H)_{-1}^{+}$inherited from the unique Hausdorff linear topology on $B_{s}(H)$ (we recall that $H$ is supposed to be finite dimensional).

Below we shall see that the situation is much different with the $\odot-$ automorphisms of the larger structure $B(H)^{+}$. There are much less continuous $\odot$-automorphisms and they are all of a nice compact form.

Let us begin with the necessary definitions and a few important facts. For any $A, B \in B(H)^{+}$, the logarithmic product $A \odot B$ is defined by

$$
A \odot B=\lim _{n \rightarrow \infty}\left(A^{1 / n} B^{1 / n}\right)^{n} .
$$

To see that the above sequence is convergent and its limit is a positive semidefinite operator, we argue as follows. For any $A \in B_{s}(H)$ let $\operatorname{supp} A$ stand for the orthogonal complement of the kernel of $A$ (which equals the range of $A$ ). For any $A \in B(H)^{+}$denote by $\log _{+} A$ the logarithm of the restriction of $A$ to $\operatorname{supp} A$ (that restriction of $A$ is a positive definite operator hence its logarithm exists). Observe that in the matrix representation of operators with respect to an orthonormal basis, if $A$ is a diagonal matrix, then $\log _{+} A$ is a diagonal matrix (of possibly smaller size) where the function $\log$ is applied to all nonzero diagonal entries of the matrix of $A$. If $X$ is a linear operator from a subspace $M$ of $H$ into $M$ and $P_{M}$ denotes the orthogonal projection of $H$ onto $M$, then $P_{M} \exp (X) P_{M}$ is a well-defined linear operator on $H$ which acts as $\exp (X)$ on $M$ and zero on $M^{\perp}$. In what follows we denote this operator by $e^{X}$. Clearly, for every $A \in B(H)^{+}$and 
positive integer $n$ we have

$$
e^{\frac{1}{n} \log _{+} A}=P_{\operatorname{supp} A}\left(\exp \left(\frac{1}{n} \log _{+} A\right)\right) P_{\operatorname{supp} A}=A^{1 / n}
$$

Let $X, Y$ be positive semidefinite operators acting on the subspaces $M, N$ of $H$ respectively, and let $P$ stand for the orthogonal projection onto $M \cap N$. Define the operator $X \dot{+} Y$ on $M \cap N$ by

$$
(X \dot{+} Y) h=P(X h+Y h) .
$$

Clearly, $X \dot{+} Y$ is a positive semidefinite operator on $M \cap N$. According to a generalization of the famous Trotter's product formula due to Kato (see p. 193 in [5], or Theorem 1.2 on p. 6 in [7]) we have

$$
\lim _{n \rightarrow \infty}\left(e^{-t X / n} e^{-t Y / n}\right)^{n}=e^{-t(X \dot{+} Y)}
$$

for every real number $t>0$. Kato's result is about spaces of arbitrary (not necessarily finite) dimensions and the limit is originally meant in the strong operator topology. But as in our case $H$ is finite dimensional, this limit is the same as the limit in the unique Hausdorff linear topology on $B(H)$.

Supposing $A, B \in B(H)^{+}$and $A, B \leq I$, we have that $-\log _{+} A,-\log _{+} B$ are positive semidefinite operators acting on $\operatorname{supp} A$ and $\operatorname{supp} B$, respectively. Applying the generalized product formula (1) for $X=-\log _{+} A$, $Y=-\log _{+} B, t=1$ and denoting $P_{\text {supp } A \cap \operatorname{supp} B}$ by $P_{A, B}$, we have

$$
\begin{aligned}
& \lim _{n \rightarrow \infty}\left(A^{1 / n} B^{1 / n}\right)^{n}=e^{\log _{+} A \dot{+} \log _{+} B}= \\
& P_{A, B}\left(\exp \left(P_{A, B}\left(\log _{+} A\right) P_{A, B}+P_{A, B}\left(\log _{+} B\right) P_{A, B}\right)\right) P_{A, B} .
\end{aligned}
$$

Therefore, $\lim _{n \rightarrow \infty}\left(A^{1 / n} B^{1 / n}\right)^{n}$ exists if $A, B \in B(H)^{+}$are such that $A, B \leq$ $I$. In the general case we can multiply $A$ and $B$ by certain positive numbers to obtain operators which are less than or equal to $I$ and the existence of the limit of the sequence $\left(\left(A^{1 / n} B^{1 / n}\right)^{n}\right)$ follows from the previous case.

Below we collect a few important properties of the logarithmic product that can be checked easily:

(i) the operation $\odot$ makes $B(H)^{+}$a commutative semigroup with unit $I$

(ii) for commuting $A, B \in B(H)^{+}$we have $A \odot B=A B$;

(iii) $(\lambda A) \odot B=\lambda(A \odot B)$ holds for all $A, B \in B(H)^{+}$and scalar $\lambda \geq 0$.

(iv) for any $A, B \in B(H)^{+}$we have $\operatorname{supp}(A \odot B)=\operatorname{supp} A \cap \operatorname{supp} B$.

In what follows we describe the $\odot$-automorphisms of $B(H)^{+}$under a mild continuity assumption.

Theorem. Let $\phi: B(H)^{+} \rightarrow B(H)^{+}$be an automorphism with respect to the logarithmic product, i.e., a bijective map satisfying

$$
\phi(A \odot B)=\phi(A) \odot \phi(B) \quad\left(A, B \in B(H)^{+}\right) .
$$

Suppose that $\phi$ is continuous at 0 with respect to the topology on $B(H)^{+}$ inherited from the unique Hausdorff linear topology on $B_{s}(H)$. Then there 
exists an invertible linear or conjugate-linear operator $T$ on $H$ such that $\phi$ is of the form

$$
\phi(A)=P_{A}^{T}\left(\exp \left(P_{A}^{T} T\left(\log _{+} A\right) T^{*} P_{A}^{T}\right)\right) P_{A}^{T} \quad\left(A \in B(H)^{+}\right)
$$

where $P_{A}^{T}$ denotes the orthogonal projection of $H$ onto $\left(T\left[(\operatorname{supp} A)^{\perp}\right]\right)^{\perp}$.

Remark. We remark that without assuming any sort of continuity, the conclusion in the above statement is false. To see this, for every $A \in B(H)^{+}$ consider the operator $A^{\dagger}$ which equals the inverse of $A$ on $\operatorname{supp} A$ and zero on $(\operatorname{supp} A)^{\perp}$. Obviously, $A^{\dagger}$ is just the Moore-Penrose pseudoinverse of $A$. One can check that the transformation $A \mapsto A^{\dagger}$ is an $\odot$-automorphism of $B(H)^{+}$which is not continuous at the singular elements of $B(H)^{+}$and is not of the form (3).

Proof of the theorem. Let us denote the (finite) dimension of $H$ by $m$. We begin the proof with some easy and useful observations. Clearly, an element $A \in B(H)^{+}$is a projection if and only if $A \odot A=A$. It follows that $\phi$ preserves the projections meaning that an operator $A \in B(H)^{+}$is a projection if and only if $\phi(A)$ is a projection.

For any projections $P, Q$ on $H$, let $P \wedge Q$ denote the infimum of the set $\{P, Q\}$ in the lattice of all projections on $H(P \wedge Q$ equals the projection of $H$ onto the intersection of the ranges of $P$ and $Q$ ). Using (2) one can check that $P \odot Q=P \wedge Q$. We easily deduce that $\phi$ is a lattice automorphism of the set of all projections. In particular, $\phi$ preserves the rank of projections, and we have $\phi(0)=0, \phi(I)=I$.

By the property (iv) above, for any $A, B \in B(H)^{+}$we have $A \odot B=0$ if and only if $\operatorname{supp} A \cap \operatorname{supp} B=\{0\}$. Therefore, we obtain that $\operatorname{supp} A \cap$ $\operatorname{supp} B=\{0\}$ if and only if $\operatorname{supp} \phi(A) \cap \operatorname{supp} \phi(B)=\{0\}$. As we know that $\phi$ preserves the rank of projections, now it easily follows that $\phi$ preserves the rank of arbitrary operators, too.

We shall need the following useful formula: for any $A \in B(H)^{+}$and rank-one projection $P$ we have

$$
A \odot P= \begin{cases}e^{\operatorname{tr}\left(\left(\log _{+} A\right) P\right)} P, & \text { if supp } P \subset \operatorname{supp} A ; \\ 0, & \text { otherwise. }\end{cases}
$$

In fact, this follows from the property (iv) of the logarithmic product in the case when $\operatorname{supp} P$ is not contained in $\operatorname{supp} A$. If $\operatorname{supp} P \subset \operatorname{supp} A$, then by (2) we infer $A \odot P=P\left(\exp \left(P\left(\log _{+} A\right) P\right)\right) P$. One can check that in the present situation we have $P\left(\log _{+} A\right) P=\left(\operatorname{tr}\left(\left(\log _{+} A\right) P\right)\right) P$ from which the formula above can be derived easily.

The essential part of the proof which now follows is divided into several steps.

Step 1. For any rank-one projection $P$ we have a bijective function $f_{P}$ : $[0, \infty[\rightarrow[0, \infty[$ such that

$$
\phi(\lambda P)=f_{P}(\lambda) \phi(P) \quad(\lambda \geq 0) .
$$


The function $f_{P}$ is multiplicative and by the continuity of $\phi$ at 0 it is of the form $f_{P}(\lambda)=\lambda^{c_{P}}(\lambda \geq 0)$ with some constant $c_{P}>0$.

To see this, let $\lambda>0$. Since $\phi(0)=0$ and $\phi$ is bijective, it follows that $\phi(\lambda P) \neq 0$. Apparently, $(\lambda P) \odot P=\lambda(P \odot P)=\lambda P$, hence $\phi(\lambda P) \odot \phi(P)=$ $\phi(\lambda P) \neq 0$. Since $\phi$ maps rank-one projections onto rank-one projections, $\phi(P)$ is also a rank-one projection with supp $\phi(P)$ being a one-dimensional subspace. Applying (iv) we have $\operatorname{supp} \phi(P) \subset \operatorname{supp} \phi(\lambda P)$. As $\phi$ preserves the rank of operators, $\phi(\lambda P)$ is of rank one, and consequently it is a scalar multiple of $\phi(P)$. We conclude that $\phi(\lambda P)=f_{P}(\lambda) \phi(P), \lambda \geq 0$ holds for some bijective function $f_{P}:\left[0, \infty\left[\rightarrow\left[0, \infty\left[\right.\right.\right.\right.$, where clearly $f_{P}(0)=0$.

Note that $(\lambda P) \odot(\mu P)=(\lambda \mu) P \odot P=(\lambda \mu) P$, hence $f_{P}(\lambda) \phi(P) \odot$ $f_{P}(\mu) \phi(P)=f_{P}(\lambda) f_{P}(\mu) \phi(P)=f_{P}(\lambda \mu) \phi(P)$, so $f_{P}$ is multiplicative. Since $\phi$ is continuous at 0 , the function $f_{P}$ is also continuous at 0 .

Let $\varphi$ be a multiplicative function from the set of all nonnegative real numbers into itself which maps positive numbers onto positive ones. Suppose that $\varphi$ is continuous at 0 . Considering the function $\log \circ \varphi \circ \exp$ we obtain an additive function on the whole set of reals which is additive and has limit $-\infty$ at $-\infty$. This clearly implies that the graph of that additive function is not dense in the plane. But any additive function with this property is necessarily a scalar multiple of the identity. Indeed, it follows, for example, from [6], Theorem 12.1.2. In our case the scalar multiple is necessarily positive and it follows that the original function $\varphi$ is a power function with positive power.

Therefore, the function $f_{P}$ is of the form $f_{P}(\lambda)=\lambda^{c_{P}}, \lambda \geq 0$ for some $c_{P}>0$.

Step 2. For any $A, B \in B(H)_{-1}^{+}$we have $\log A \leq \log B$ if and only if $\log \phi(A) \leq \log \phi(B)$. The bijective additive map $\log \circ \phi \circ \exp$ on $B_{s}(H)$ preserves the usual order among self-adjoint operators and hence it is reallinear.

Let $A, B \in B(H)_{-1}^{+}$. We have $\log A \leq \log B$ if and only if $\langle(\log A) x, x\rangle \leq$ $\langle(\log B) x, x\rangle$ holds for every unit vector $x \in H$. This is equivalent to $e^{\operatorname{tr}((\log A) P)} \leq e^{\operatorname{tr}((\log B) P)}$ and, as the function $f_{P}$ is strictly increasing, also to

$$
f_{P}\left(e^{\operatorname{tr}((\log A) P)}\right) \phi(P) \leq f_{P}\left(e^{\operatorname{tr}((\log B) P)}\right) \phi(P)
$$

for every rank-one projection $P$. Using (4) we compute

$$
f_{P}\left(e^{\operatorname{tr}((\log A) P)}\right) \phi(P)=\phi\left(e^{\operatorname{tr}((\log A) P)} P\right)=\phi(A \odot P)=\phi(A) \odot \phi(P)
$$

and similarly have

$$
f_{P}\left(e^{\operatorname{tr}((\log B) P)}\right) \phi(P)=\phi(B) \odot \phi(P) .
$$

Applying formula (4) we see that

$$
\phi(A) \odot \phi(P) \leq \phi(B) \odot \phi(P)
$$

holds for every rank-one projection $P$ if and only if $\left.e^{\operatorname{tr}((\log \phi(A)) \phi(P))}\right) \leq$ $\left.e^{\operatorname{tr}((\log \phi(B)) \phi(P))}\right)$ is valid for every rank-one projection $P$. Clearly, this is 
equivalent to $\log \phi(A) \leq \log \phi(B)$. That verifies that $\log A \leq \log B$ if and only if $\log \phi(A) \leq \log \phi(B)$.

Now we show that the bijective map $\psi=\log \circ \phi \circ \exp$ preserves the order on $B_{s}(H)$. Let $A, B \in B_{s}(H)$. Then there exist $A_{1}, B_{1} \in B(H)_{-1}^{+}$such that $A=\log A_{1}$ and $B=\log B_{1}$. We infer that $A=\log A_{1} \leq \log B_{1}=B$ if and only if $\log \phi(\exp (A))=\log \phi\left(A_{1}\right) \leq \log \phi\left(B_{1}\right)=\log \phi(\exp (B))$. Hence $\psi$ preserves the order.

Since $\phi$ preserves the logarithmic product, $\psi$ is additive on $B_{s}(H)$. In fact, this follows from

$$
\begin{aligned}
& \psi(A+B)=\log \left(\phi\left(e^{A+B}\right)\right)=\log \left(\phi\left(e^{A} \odot e^{B}\right)\right) \\
& =\log \left(\phi\left(e^{A}\right) \odot \phi\left(e^{B}\right)\right)=\log \phi\left(e^{A}\right)+\log \phi\left(e^{B}\right)=\psi(A)+\psi(B) .
\end{aligned}
$$

Hence $\psi(0)=0$ and $\psi(q A)=q \psi(A)$ for every rational number $q$. Let $A \in B(H)^{+}$and $\lambda>0$. We see that $\lambda A$ is the infimum of the set of all operators of the form $q A$ where $q \geq \lambda$ is rational number. As $\psi$ preserves the order, it follows that $\psi(\lambda A)$ is the infimum of the set of all $\psi(q A)=$ $q \psi(A)$ which infimum is, on the other hand, equal to $\lambda \psi(A)$. We obtain $\psi(\lambda A)=\lambda \psi(A)$ and this gives us that $\psi$ is positive homogenous on $B(H)^{+}$. By the additivity of $\psi$ it easily follows that $\psi$ is homogeneous on $B_{s}(H)$.

Step 3. The structure of all linear order automorphisms of $B_{s}(H)$ is well-known. One can refer, for example, to Corollary 5 in [4] and deduce that $\log \circ \phi \circ \exp$ being such a transformation, it is necessarily of the form $S \mapsto T S T^{*}$ with some nonsingular linear or conjugate-linear operator $T$ on $H$. It follows that

$$
\phi(A)=\exp \left(T(\log A) T^{*}\right) \quad\left(A \in B(H)_{-1}^{+}\right) .
$$

Our final aim is to extend the formula (5) to cover all elements of $B(H)^{+}$. We proceed as follows. For a unit vector $x \in H$ we denote by $P_{x}$ the orthogonal projection onto the subspace generated by $x$.

Step 4. For any pair $x \in H$ and $y \in\left(T\left(x^{\perp}\right)\right)^{\perp}$ of unit vectors we have $\phi\left(P_{x}\right)=P_{y}$.

To see this, let $x \in H$ be a unit vector. Set $Q_{x}=I-P_{x}$. For any $\lambda>0$ we have $P_{x}+\lambda Q_{x} \in B(H)_{-1}^{+}$, so by the previous step $\phi\left(P_{x}+\lambda Q_{x}\right)=$ $\exp \left(T\left(\log \left(P_{x}+\lambda Q_{x}\right)\right) T^{*}\right)$. Observe that $\log \left(P_{x}+\lambda Q_{x}\right)=(\log \lambda) Q_{x}$, so we have $\log \left(P_{x}+\lambda Q_{x}\right) x=0$.

Define $y=\frac{\left(T^{*}\right)^{-1} x}{\left\|\left(T^{*}\right)^{-1} x\right\|}$. Then we obtain $T \log \left(P_{x}+\lambda Q_{x}\right) T^{*} y=T \log \left(P_{x}+\right.$ $\left.\lambda Q_{x}\right) \frac{x}{\left\|\left(T^{*}\right)^{-1} x\right\|}=0$. If for any operator $A \in B(H)$ and vector $u \in H$ we have $A u=0$, then $(\exp A) u=u$. This follows from the Taylor series expansion of the exponential function. Applying this observation, we infer $\phi\left(P_{x}+\lambda Q_{x}\right) y=e^{T \log \left(P_{x}+\lambda Q_{x}\right) T^{*}} y=y$ for any $\lambda>0$.

Next we show for any $z \in y^{\perp}$ that $\phi\left(P_{x}+\lambda Q_{x}\right) z \rightarrow 0$ as $\lambda \rightarrow 0$. Let $0<\lambda<1$. Consider the spectral resolution of the positive semidefinite operator $T Q_{x} T^{*}$. Since it is of rank $m-1$, there are mutually orthogonal rank-one projections $R_{2}, \ldots, R_{m}$ and positive numbers $\mu_{2}, \ldots, \mu_{m}$ such that 
$T Q_{x} T^{*}=\sum_{k=2}^{m} \mu_{k} R_{k}$. Denote $R_{1}=I-\sum_{k=2}^{m} R_{k}$. We compute

$$
\begin{aligned}
\phi\left(P_{x}+\lambda Q_{x}\right)=e^{T \log \left(P_{x}+\lambda Q_{x}\right) T^{*}}= & e^{(\log \lambda) T Q_{x} T^{*}}=e^{(\log \lambda) \sum_{k=2}^{m} \mu_{k} R_{k}} \\
& =e^{\sum_{k=2}^{m}\left(\log \lambda^{\mu_{k}}\right) R_{k}}=R_{1}+\sum_{k=2}^{m} \lambda^{\mu_{k}} R_{k} .
\end{aligned}
$$

We know that $\phi\left(P_{x}+\lambda Q_{x}\right) y=y$. Since all $\lambda^{\mu_{k}}$ are less than 1 , we deduce that $y$ is in the range of the rank-one projection $R_{1}$. If $z \in y^{\perp}$, then it follows that $R_{1} z=0$ and hence we obtain

$$
\phi\left(P_{x}+\lambda Q_{x}\right) z=\sum_{k=2}^{m} \lambda^{\mu_{k}} R_{k} z
$$

and this converges to 0 as $\lambda \rightarrow 0$. Therefore, we conclude that

$$
\lim _{\lambda \rightarrow 0} \phi\left(P_{x}+\lambda Q_{x}\right)=P_{y}
$$

holds in the strong operator topology (topology of pointwise convergence). Since the underlying Hilbert space is finite dimensional, we obtain that the above convergence holds also in the operator norm topology.

For temporary use denote $A_{\lambda}=\phi\left(P_{x}+\lambda Q_{x}\right)$ for any $\lambda>0$. We show that $\lim _{\lambda \rightarrow 0} A_{\lambda}=P_{y}$ implies that $\lim _{\lambda \rightarrow 0} A_{\lambda} \odot P_{u}=0$ for every unit vector $u \in H$ with $P_{u} \neq P_{y}$. To verify this, observe that by (4) we have $A_{\lambda} \odot$ $P_{u}=e^{\operatorname{tr}\left(\left(\log A_{\lambda}\right) P_{u}\right)} P_{u}$. Consequently, we have to show that $\operatorname{tr}\left(\left(\log A_{\lambda}\right) P_{u}\right)=$ $\left\langle\left(\log A_{\lambda}\right) u, u\right\rangle \rightarrow-\infty$ as $\lambda \rightarrow 0$. In order to see this, pick a unit vector $u \in H$ such that $P_{u} \neq P_{y}$. It follows that $u$ and $y$ are linearly independent, hence $|\langle u, y\rangle|<1$ which implies $\|u\|^{2}-|\langle u, y\rangle|^{2}>0$. Take an arbitrary real number $K$. Choose $\epsilon>0$ such that $(\log (1+\epsilon))|\langle u, y\rangle|^{2}+(\log \epsilon)\left(\|u\|^{2}-|\langle u, y\rangle|^{2}\right)<K$. We know that there exists a $\lambda_{0}>0$ such that $\left\|A_{\lambda}-P_{y}\right\| \leq \epsilon$ holds if $0<\lambda<\lambda_{0}$. Since $A_{\lambda}-P_{y}$ is self-adjoint, we infer $-\epsilon I \leq A_{\lambda}-P_{y} \leq \epsilon I$ for all $0<\lambda<\lambda_{0}$. It is well known that the logarithm is an operator monotone function. Using this fact we deduce $\log A_{\lambda} \leq \log \left(P_{y}+\epsilon I\right)$ and hence we obtain that

$$
\begin{gathered}
\left\langle\left(\log A_{\lambda}\right) u, u\right\rangle \leq\left\langle\left(\log \left(P_{y}+\epsilon I\right) u, u\right\rangle=\left\langle\left(\log (1+\epsilon) P_{y}+(\log \epsilon)\left(I-P_{y}\right)\right) u, u\right\rangle\right. \\
=(\log (1+\epsilon))|\langle u, y\rangle|^{2}+(\log \epsilon)\left(\|u\|^{2}-|\langle u, y\rangle|^{2}\right)<K
\end{gathered}
$$

holds for every $0<\lambda<\lambda_{0}$. This shows that $\left\langle\left(\log A_{\lambda}\right) u, u\right\rangle \rightarrow-\infty$ as $\lambda \rightarrow 0$ and hence that $\lim _{\lambda \rightarrow 0} A_{\lambda} \odot P_{u}=0$ holds for every unit vector $u \in H$ with $P_{u} \neq P_{y}$.

Assume that $\phi\left(P_{x}\right) \neq P_{y}$. Then by the above we have $\phi\left(P_{x}+\lambda Q_{x}\right) \odot$ $\phi\left(P_{x}\right) \rightarrow 0$ as $\lambda \rightarrow 0$. However, on the other hand, as $P_{x}+\lambda Q_{x}, P_{x}$ commute, we see that $\phi\left(P_{x}+\lambda Q_{x}\right) \odot \phi\left(P_{x}\right)=\phi\left(\left(P_{x}+\lambda Q_{x}\right) \odot P_{x}\right)=\phi\left(\left(P_{x}+\lambda Q_{x}\right) P_{x}\right)=$ $\phi\left(P_{x}\right)$ for every $\lambda>0$. Since this is an obvious contradiction, it follows that $\phi\left(P_{x}\right)=P_{y}$ is true for $y=\frac{\left(T^{*}\right)^{-1} x}{\left\|\left(T^{*}\right)^{-1} x\right\|}$. Clearly, $T^{*} y$ belongs to the linear span of $x$ which implies that $y \in\left(T\left(x^{\perp}\right)\right)^{\perp}$. Since $T$ is invertible, $\left(T\left(x^{\perp}\right)\right)^{\perp}$ 
is one-dimensional, so for every unit vector $v$ from this subspace we have $P_{v}=P_{y}$. This completes the proof of Step 4 .

Next we determine the transformation $\phi$ on the rank-one elements of $B(H)^{+}$.

Step 5. Keeping the notation in Step 4, we have

$$
\phi\left(\lambda P_{x}\right)=\lambda^{\left\|T^{*} y\right\|^{2}} P_{y} \quad(\lambda \geq 0) .
$$

If $\lambda=0$, this is trivial. Let $\lambda>0$. We have $\phi(\lambda I)=e^{T(\log \lambda) I T^{*}}=$ $e^{(\log \lambda) T T^{*}}$. Applying formula (4), it follows that

$$
\begin{aligned}
& \phi\left(\lambda P_{x}\right)=\phi\left((\lambda I) \odot P_{x}\right)=\phi(\lambda I) \odot P_{y} \\
&=e^{\operatorname{tr}\left((\log \lambda) T T^{*} P_{y}\right)} P_{y}=e^{(\log \lambda)\left\langle T T^{*} y, y\right\rangle} P_{y}=\lambda^{\left\|T^{*} y\right\|^{2}} P_{y} .
\end{aligned}
$$

In what follows we determine the transformation $\phi$ on an arbitrary element $A \in B(H)^{+}$. To do this, we first consider the support of $\phi(A)$.

Step 6. For any $A \in B(H)^{+}$we have $\operatorname{supp} \phi(A)=\left(T\left[(\operatorname{supp} A)^{\perp}\right]\right)^{\perp}$.

Let $x \in \operatorname{supp} A,\|x\|=1$. As before, let $P_{x}$ be the rank-one projection onto the subspace generated by $x$. Clearly, supp $P_{x} \subset \operatorname{supp} A$ and hence $A \odot P_{x} \neq 0$. By Step 4 it follows that $\phi(A) \odot P_{y} \neq 0$ for any $y \in\left(T\left(x^{\perp}\right)\right)^{\perp}$. Therefore $y \in \operatorname{supp} \phi(A)$ and hence $\left(T\left(x^{\perp}\right)\right)^{\perp} \subset \operatorname{supp} \phi(A)$ holds for every $x \in \operatorname{supp} A$. This easily yields $\left(T\left[\left(\operatorname{supp} A^{\perp}\right)\right]\right)^{\perp} \subset \operatorname{supp} \phi(A)$.

Let $y \in \operatorname{supp} \phi(A),\|y\|=1$. By the surjectivity of $\phi$ there exists $x \in$ $H,\|x\|=1$ such that $\phi\left(P_{x}\right)=P_{y}$ and $y \in\left(T\left(x^{\perp}\right)\right)^{\perp}$ by Step 4. Since $\phi(A) \odot P_{y} \neq 0$ and therefore $A \odot P_{x} \neq 0$, it follows that $x \in \operatorname{supp} A$. This implies that $y \in\left(T\left(x^{\perp}\right)\right)^{\perp} \subset\left(T\left[(\operatorname{supp} A)^{\perp}\right]\right)^{\perp}$. Consequently, we have $\operatorname{supp} \phi(A) \subset\left(T\left[(\operatorname{supp} A)^{\perp}\right]\right)^{\perp}$ and hence the statement in Step 6 is verified.

To complete the proof assume that $y \in \operatorname{supp} \phi(A)=\left(T\left[(\operatorname{supp} A)^{\perp}\right]\right)^{\perp}$, $\|y\|=1$. Then for $x=\frac{T^{*} y}{\left\|T^{*} y\right\|}$ we have $x \in \operatorname{supp} A,\|x\|=1$, and $\phi\left(P_{x}\right)=P_{y}$ by Step 4. Using formula (4) we have

$$
A \odot P_{x}=e^{\operatorname{tr}\left(\left(\log _{+} A\right) P_{x}\right)} P_{x} .
$$

Applying $\phi$ to the left side of this equation, we obtain, again by formula (4), that

$$
\phi\left(A \odot P_{x}\right)=\phi(A) \odot P_{y}=e^{\operatorname{tr}\left(\left(\log _{+} \phi(A)\right) P_{y}\right)} P_{y},
$$

while applying $\phi$ to the right side of (6) we obtain by Step 5 that

$$
\phi\left(e^{\operatorname{tr}\left(\left(\log _{+} A\right) P_{x}\right)} P_{x}\right)=e^{\left\|T^{*} y\right\|^{2} \operatorname{tr}\left(\left(\log _{+} A\right) P_{x}\right)} P_{y} .
$$

Since $\operatorname{tr}\left(\left(\log _{+} \phi(A)\right) P_{y}\right)=\left\langle\left(\log _{+} \phi(A)\right) y, y\right\rangle$ and $\left\|T^{*} y\right\|^{2} \operatorname{tr}\left(\left(\log _{+} A\right) P_{x}\right)=$ $\left\|T^{*} y\right\|^{2}\left\langle\left(\log _{+} A\right) x, x\right\rangle=\left\langle\left(\log _{+} A\right) T^{*} y, T^{*} y\right\rangle$, it follows

$$
\left\langle\left(\log _{+} \phi(A)\right) y, y\right\rangle=\left\langle\left(\log _{+} A\right) T^{*} y, T^{*} y\right\rangle=\left\langle T\left(\log _{+} A\right) T^{*} y, y\right\rangle
$$

for every $y \in \operatorname{supp} \phi(A)$. Let $P_{A}^{T}$ denote the orthogonal projection of $H$ onto $\operatorname{supp} \phi(A)=\left(T\left[(\operatorname{supp} A)^{\perp}\right]\right)^{\perp}$. By $(7)$ it follows that

$$
P_{A}^{T}\left(\log _{+} \phi(A)\right) P_{A}^{T}=P_{A}^{T}\left(T\left(\log _{+} A\right) T^{*}\right) P_{A}^{T} .
$$


One can check that $\phi(A)=P_{A}^{T}\left(\exp \left(P_{A}^{T}\left(\log _{+} \phi(A)\right) P_{A}^{T}\right)\right) P_{A}^{T}$ and hence we obtain

$$
\phi(A)=P_{A}^{T}\left(\exp \left(P_{A}^{T} T\left(\log _{+} A\right) T^{*} P_{A}^{T}\right)\right) P_{A}^{T}
$$

for any $A \in B(H)^{+}$. The proof of the theorem is complete.

Open problem. Applying careful considerations and computations, one can show that any nonsingular linear or conjugate-linear operator $T$ on $H$ induces an $\odot$-automorphism of $B(H)^{+}$via the formula in (3). Moreover, this automorphisms is continuous at zero. We have previously mentioned that the Moore-Penrose inverse as an operation on $B(H)^{+}$also provides an $\odot$-automorphism which is not continuous at zero. It would be interesting to know and we leave it as on open problem if any automorphism of $B(H)^{+}$ (continuous or not) can be obtained from those two kinds of automorphisms by composition.

\section{REFERENCES}

1. M. Alexa, Linear combination of transformations, In SIGGRAPH'02: Proceedings of the 29th annual conference on computer graphics and interactive techniques, ACM Press, New York, 2002, pp. 380-387.

2. V. Arsigny, P. Fillard, X. Pennec and N. Ayache, Log-Euclidean metrics for fast and simple calculus on diffusion tensors export, Magnetic Resonance in Medicine, 56 (2006), 411-421.

3. V. Arsigny, P. Fillard, X. Pennec and N. Ayache, Geometric means in a noval vector space structure on symmetric positive-definite matrices, SIAM. J. Matrix Anal. Appl. 29 (2006), 328-347.

4. R.V. Kadison, A generalized Schwarz inequality and algebraic invariants for operator algebras, Ann. of Math. 56 (1952), 494-503.

5. T. Kato, Trotter's product formula for an arbitrary pair of self-adjoint contraction semigroups, In: Topics in Functional Analysis, I. Gohberg, M. Kac (eds.), Academic Press, New York, 1978, pp. 185-195.

6. M. Kuczma (A. Gilányi (Ed.)) An Introduction to the Theory of Functional Equations and Inequalities: Cauchy's Equation and Jensen's Inequality, Birkhäuser, 2009.

7. B. Simon, Functional Integration and Quantum Physics, Academic Press, New York, 1979.

8. M.K. Warmuth and D. Kuzmin, Bayesian generalized probability calculus for density matrices, Mach. Learn. 78 (2010), 63-101.

Faculty of Electrical Engineering, University of Ljubluana, TržašKa 25, SI-1000, LuUbluana, Slovenia

Institute of Mathematics, University of Debrecen, P.O. Box 12, H-4010 Debrecen, Hungary

E-mail address: gregor.dolinar@fe.uni-lj.si, molnarl@science.unideb.hu

URL: http://www. math.unideb.hu/ ${ }^{\sim m o l n a r l / ~}$ 